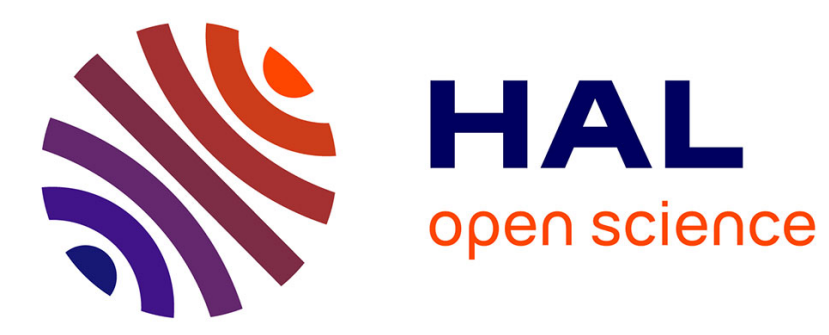

\title{
Optimal control of multiple magnetic microbeads navigating in microfluidic channels
}

Lyès Mellal, David Folio, Karim Belharet, Antoine Ferreira

\section{To cite this version:}

Lyès Mellal, David Folio, Karim Belharet, Antoine Ferreira. Optimal control of multiple magnetic microbeads navigating in microfluidic channels. IEEE International Conference on Robotics and Automation (ICRA 2016), May 2016, Stockholm, Sweden. pp.1921-1926, 10.1109/ICRA.2016.7487338 . hal-01342018

\section{HAL Id: hal-01342018 https://hal.science/hal-01342018}

Submitted on 5 Jul 2016

HAL is a multi-disciplinary open access archive for the deposit and dissemination of scientific research documents, whether they are published or not. The documents may come from teaching and research institutions in France or abroad, or from public or private research centers.
L'archive ouverte pluridisciplinaire HAL, est destinée au dépôt et à la diffusion de documents scientifiques de niveau recherche, publiés ou non, émanant des établissements d'enseignement et de recherche français ou étrangers, des laboratoires publics ou privés. 


\title{
Optimal Control of Multiple Magnetic Microbeads Navigating in Microfluidic Channels
}

\author{
Lyès Mellal $^{1}$, David Folio ${ }^{1}$, Karim Belharet ${ }^{2}$, and Antoine Ferreira ${ }^{1}$
}

\begin{abstract}
This paper presents an optimal control strategy for navigation of multiple magnetic microbeads for future drug targeting applications. To transport the drugs, we use therapeutic magnetic microbeads as navigable agents controlled by magnetic gradients. The main difficulty is to control independently each therapeutic agent along a trajectory with the same magnetic gradient fields. This study proposes an optimal control methodology to control a group of different therapeutic agents at desired states. Based on a dynamic model of group of magnetic microbeads, controllability and observability conditions are formulated and simulated. Based on the proposed theoretical analysis a linear quadratic with integral action control (LQI) has been chosen to be applied to the microbeads system. Finally, an experimental investigation is carried out in millimeter-sized fluidic artery vessels to demonstrate the controllability and stability of two magnetic microbeads under different velocity and trajectory constraints with a laminar viscous fluidic environment.
\end{abstract}

\section{INTRODUCTION}

To enhance the targeted therapy against the cancer and avoid the damage in the tissue, closed-loop control is necessary for computerized drug delivery of therapeutic agents. A promising approach is to use swarms or agglomerates of untethered magnetic microrobots -coated with therapeutic drugs- controlled by external magnetic gradient fields provide by a magnetic resonance imaging (MRI)scanner or electromagnetic systems. These microrobots are commonly termed therapeutic micro carriers (TMMC) [1]. In such settings, one of the main obstacles hindering the development of automated drug delivery TMMCs is the lack of accurate mathematical models describing the dynamic effects for robust and stable navigation control of multiple TMMCs interacting mutually [2]. Some previous works have considered the magnetic control of single magnetic microrobots with linear [3] and nonlinear [4] formulations of their dynamic motion, and more recently, preliminary studies have been conducted on the magnetic control of multiple microrobots [5], [6]. Theses studies point out the position control problem since the group of magnetic microrobots are exposed to the same control input: the magnetic gradient field.

In [6], the authors have investigated the control of geometrically dissimilar Mag- $\mu$ Bots and a group of identicallyfabricated Mag- $\mu$ Bots in a free environment. In [5], the authors demonstrated theoretically the closed-loop control of a group of millimeter-scale particles immersed in fluid and

\footnotetext{
${ }^{1}$ L. Mellal, D. Folio and A. Ferreira are with INSA Centre Val de Loire, Université d'Orléans, PRISME EA 4229, Bourges, France. Corresponding author: Antoine Ferreira (Email: antoine.ferreira@insa-cvl.fr

${ }^{2} \mathrm{~K}$.Belharet is with Hautes Études d'Ingénieur campus Centre, PRISME EA 4229, Châteauroux, France.
}

driven by the MRI scanner. As a result, the authors proved through simulation results the stability of two millimetersized beads at the desired positions. However, experimental validation of the proposed controllability and stability conditions in operating conditions are still undergoing. The main contribution of this study is to apply optimal control methodology for the position stabilization of two geometrically similar/dissimilar microbeads at the desired states in a microfluidic vascular channel mimicking the blood flow arterial network. A mathematical model of a group of $n$ magnetic microrobots is proposed taking into account the main magnetic, hydrodynamic and interaction microforces. As the multi-robotic system behaves as a underactuated dynamic system, controllability and observability conditions are formulated in order to demonstrate the robust stability. A linear quadratic with integral action control (LQI) has been chosen taking into account the simplicity of its implementation in the position control of multiple robotic systems. Also, it presents good performance in position stabilization and robustness. As example, we can cite the attitude and positioning control of small scale unmanned helicopter [7], the motion and vibration control of a three dimensional flexible shaking table [8] or electromagnetic navigating tunneling robots [9]. Finally, experimental results demonstrate the optimal stabilization of the 1D positions of two magnetic microbeads navigating in a microfluidic channel.

\section{Mathematical Modeling}

To enhance the targeted therapy efficiency, this work aims to control a group of magnetic microbeads. The magnetic control of such system is challenging since it is underactuated, i.e, all microbeads are subjected to the same input: the magnetic gradient field $\nabla \mathbf{b}$.

\section{A. Magnetic and Hydrodynamic Forces}

Let consider $n$ magnetic microbeads that are injected in a microfluidic environment, as shown in Fig. 1. The microbeads are considered as point magnetic dipoles separated by a distance vector $\mathbf{d}_{i j}$. When a magnetic gradient field $\nabla \mathbf{b}$ is applied, a magnetic force is exerted on the magnetic spherical volume ${ }^{i} V$ of the microbead $i=1, \ldots, n$, and is basically expressed as:

$$
{ }^{i} \mathbf{f}_{\mathrm{m}}={ }^{i} V\left({ }^{i} \mathbf{M} \cdot \nabla\right) \mathbf{b}
$$

where $\mathbf{b}=\left(b_{x}, b_{y}, b_{z}\right)^{T}$ is the magnetic field; and ${ }^{i} \mathbf{M}$ the magnetization of the $i$ th dipole. In this study, hard magnetic microbeads are addressed (such as neodymium magnet, 
$\mathrm{NdFeB}$ ), and their dipoles are considered magnetized at the saturation, ${ }^{i} \mathbf{M}=\left({ }^{i} M_{\text {sat }},{ }^{i} M_{\text {sat }},{ }^{i} M_{\text {sat }}\right)^{T}$. Secondly, when the magnetic and gradient fields are generated from respectively Helmholtz and Maxwell coils, the cartesian components of the magnetic force (1) can be rewritten as:

$$
\left[\begin{array}{l}
{ }^{i} f_{m x} \\
{ }^{i} f_{m y} \\
{ }^{i} f_{m z}
\end{array}\right]={ }^{i} V^{i} M_{\text {sat }}\left[\begin{array}{l}
\frac{\partial b_{x}}{\partial x} \\
\frac{\partial b_{y}}{\partial y} \\
\frac{\partial b_{z}}{\partial z}
\end{array}\right]
$$

When the magnetic microbeads move in a microfluidic environment, their dynamics are mainly counteracted by a drag force. Considering the creeping flow of an incompressible Newtonian fluid, the drag force exerted on a spherical device is estimated from Stoke's law [10]:

$$
{ }^{i} \mathbf{f}_{\mathrm{d}}=-6 \pi \eta_{f}{ }^{i} r^{i} \mathbf{v}
$$

with $\eta_{f}$ the fluid viscosity; ${ }^{i} r$ and ${ }^{i} \mathbf{v}$ respectively the radii and velocities of the $i$ th microbead.

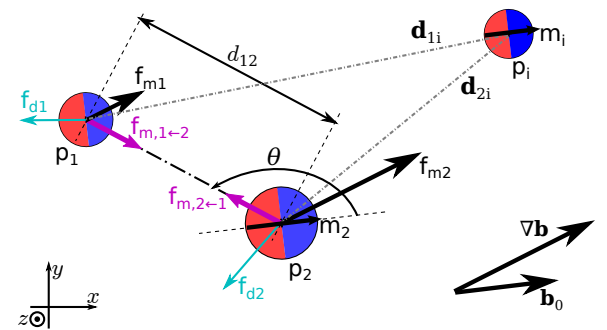

Fig. 1. Group of magnetic microbeads in a microfluidic environment.

\section{B. Separation Distance}

Commonly, when several magnetic microbeads are in a closed range, a magnetic interaction occurs [11]. Specifically, a magnetic microbead located at $\mathbf{p}_{i}=\left(x_{i}, y_{i}, z_{i}\right)^{T}$ with its dipole moment ${ }^{i} \mathbf{m}={ }^{i} \mathbf{M}{ }^{i} V$ induces a magnetic field in location $\mathbf{p}$ given by [12]:

$$
\mathbf{b}_{\mathbf{p}_{i}}(\mathbf{p})=\frac{\mu_{0}}{4 \pi}\left(3\left({ }^{i} \mathbf{m} \cdot \mathbf{d}_{0}\right) \mathbf{d}_{0}-{ }^{i} \mathbf{m}\right)
$$

where $\mu_{0}=4 \pi \times 10^{-7} \mathrm{Tm} / \mathrm{A}$ is the vacuum permeability; $\mathbf{d}_{0}=\frac{\left(\mathbf{p}-\mathbf{p}_{i}\right)}{\left\|\mathbf{p}-\mathbf{p}_{i}\right\|}$ is the separation distance unit vector. Thus, the force acting on the magnetic microbead 2 (dipole moment ${ }^{2} \mathbf{m}$ ) due to the presence of a microbead 1 (dipole moment ${ }^{1} \mathbf{m}$ ) is expressed as [13]:

$$
\begin{aligned}
\mathbf{f}_{\mathrm{m}, 2 \leftarrow 1}=\frac{3 \mu_{0}}{4 \pi d_{12}^{4}} & \left(\mathbf{d}\left({ }^{1} \mathbf{m} \cdot{ }^{2} \mathbf{m}\right)+{ }^{1} \mathbf{m}\left(\mathbf{d}_{12} \cdot{ }^{2} \mathbf{m}\right)\right. \\
+ & { }^{2} \mathbf{m}\left(\mathbf{d}_{12} \cdot{ }^{1} \mathbf{m}\right) \\
& \left.-5 \frac{\mathbf{d}}{d_{12}^{2}}\left(\mathbf{d}_{12} \cdot{ }^{2} \mathbf{m}\right)\left(\mathbf{d}_{12} \cdot{ }^{1} \mathbf{m}\right)\right)
\end{aligned}
$$

where $\mathbf{d}_{12}=d_{12} \mathbf{d}_{0}$ is the separation distance vector. Obviously, the Newton's third law implies: $\mathbf{f}_{\mathrm{m}, 1 \leftarrow 2}=-\mathbf{f}_{\mathrm{m}, 2 \leftarrow 1}$. Then, the magnetic force interaction magnitude is expressed as:

$$
{ }^{12} f_{m i}=\frac{3 \mu_{0}\left\|\mathbf{m}_{1} \mathbf{m}_{2}\right\|}{4 \pi d^{4}} \sqrt{1-2 \cos ^{2} \theta_{12}+5 \cos ^{4} \theta_{12}}
$$

with $\theta_{12}$ the angle between the dipole moment $\mathbf{m}_{j}(j=1$ or 2) and the separation distance direction $\mathbf{d}_{12}$ (see Fig. 1). First, we assumed that all dipole moments of the microbeads (such as neodymium magnet, $\mathrm{NdFeB}$ ) are aligned along the uniform field $\mathbf{b}_{0}$ and are saturated to the maximum value $\mathbf{m}_{j}={ }^{j} \mathbf{M}_{\mathrm{sat}}{ }^{j} V$. Secondly, for a given separation distance $d_{12}$ the above interaction force magnitude is minimal for $\theta_{\min }=k 180^{\circ}+\left\{63.43^{\circ}, 116.56^{\circ}\right\}$, and maximal for $\theta_{\max }=$ $k 180^{\circ}+\left\{0,180^{\circ}\right\}, \forall k \in \mathbb{Z}$. Commonly, the magnetic gradient is limited by the capability of the magnetic coils system. Hence, the minimal controllable separation distance is given by:

$$
d_{\min }=\left(\frac{6 \mu_{0}\left\|\mathbf{m}_{a} \mathbf{m}_{b}\right\|}{4 \pi\left\|^{i} \mathbf{f}_{\mathrm{m} \max }\right\|}\right)^{1 / 4}
$$

with $\left\|{ }^{i} \mathbf{f}_{\text {max }}\right\|$ the maximum magnetic force induced by the magnetic coils system. Fig. 2 shows the evolution of the interaction force (6) as function of the separation distance $d$ for $\theta_{\min }$ (red dash-line) and $\theta_{\max }$ (blue dash-line). As example, for a magnetic actuation system capable to generate a magnetic gradient up to $\|\nabla \mathbf{b}\|_{\max }=1.5 \mathrm{~T} / \mathrm{m}$, the minimal controllable separation distance is settled to $d_{\min }=8.86 \mathrm{~mm}$. Finally, as the interaction force evolves in $1 / d^{4}$, above $d>10 \mathrm{~mm}$ it becomes negligible.

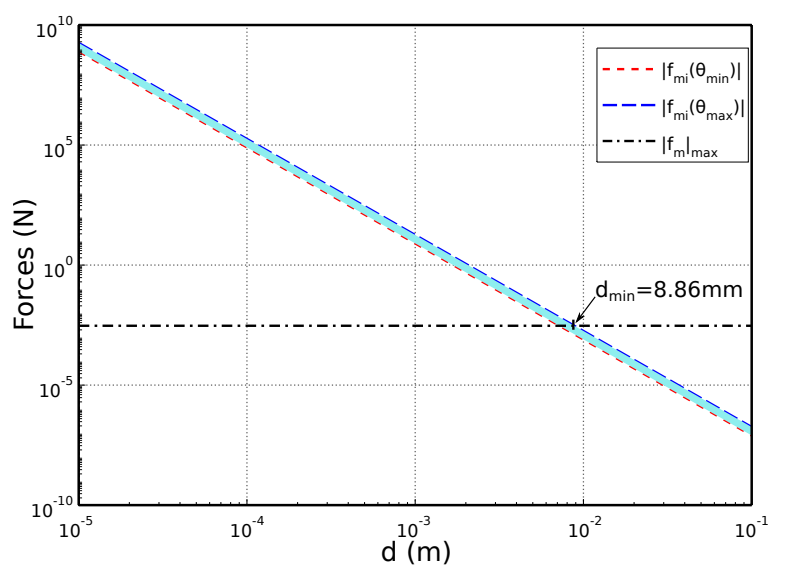

Fig. 2. Interaction forces $f_{m i}$ magnitude as function of the separation distance $d$.

\section{Control of a Group of Magnetic Microbeads}

\section{A. System Dynamics}

In this section we assume that i) the gravitational forces are considered compensated by the buoyancy forces and the sustentation magnetic force in $z$-axis; ii) the magnetic microbeads are not in contact; iii) the separation distance $d$ is large enough so that the interaction between microbeads could be neglected, that is $f_{m i} \approx 0$; and iv) the other microforces (such as van der Waals, electrostatic or steric forces) are not significant. Following the Newton's second law, the motion of the $i_{t h}$ magnetic microbead can be expressed as:

$$
{ }^{i} \rho^{i} V{ }^{i} \dot{\mathbf{v}}={ }^{i} \mathbf{f}_{\mathrm{m}}+{ }^{i} \mathbf{f}_{\mathrm{d}}
$$


with ${ }^{i} \rho$ the density of the microbead $i$. For the sake of simplicity in the remainder of this paper, only two-dimensional (2D) motions in the $x-y$ plane are considered. Thus, the 2D dynamics of each magnetic microbead is defined as follows:

$$
\left(S_{i}\right)\left\{\begin{array}{l}
\dot{x}_{i}=v_{x i} \\
\dot{v}_{x i}=\alpha_{x i} v_{x i}+\beta_{x i} \frac{\partial b_{x}}{\partial x} \\
\dot{y}_{i}=v_{y i} \\
\dot{v}_{y i}=\alpha_{y i} v_{y i}+\beta_{y i} \frac{\partial b_{y}}{\partial x}
\end{array}\right.
$$

with

$$
\begin{aligned}
\alpha_{x i} & =-\frac{9}{2} \frac{\eta_{f}}{\rho_{i} r_{i}^{2}} \operatorname{sign}\left(v_{x i}\right)<0 \\
\alpha_{y i} & =-\frac{9}{2} \frac{\eta_{f}}{\rho_{i} r_{i}^{2}} \operatorname{sign}\left(v_{y i}\right)<0 \\
\beta_{x i} & =\beta_{y i}=\beta_{i}=\frac{{ }^{i} M_{\text {sat }}}{\rho_{i}}
\end{aligned}
$$

Neglecting the interaction microforces, we assume that each single magnetic microbead has a dynamics expressed by $\left(S_{i}\right)$ that can be divided into two independent subsystems $\left(S_{x i}\right)$ and $\left(S_{y i}\right)$. In the following, the analysis is reduced to $\left(S_{x i}\right)$ since $\left(S_{y i}\right)$ can be easily deduced from the former.

Let $\mathbf{x}=\left(x_{1}, v_{x 1}, \ldots x_{i}, v_{x i}, \ldots x_{n}, v_{x n},\right)^{T}$ denotes the state vector of a group of $n$ magnetic microbeads; $\mathbf{u}=\frac{\partial b_{x}}{\partial x}$ defines the control input; and $\mathbf{y}=\left(x_{1}, \ldots x_{i}, \ldots x_{n}\right)$ is the measured output. The state-space representation of a group of $n$ magnetic microbeads is expressed as:

$$
\left\{\begin{array}{l}
\dot{\mathbf{x}}=\mathbf{A x}+\mathbf{B u} \\
\mathbf{y}=\mathbf{C x}
\end{array}\right.
$$

with the following system matrices;

$$
\begin{aligned}
\mathbf{A} & =\left[\begin{array}{cccccccc}
0 & 1 & \cdots & 0 & 0 & \cdots & 0 & 0 \\
0 & -\alpha_{1} & \cdots & 0 & 0 & \cdots & 0 & 0 \\
\vdots & & \cdots & \vdots & & \cdots & \vdots & \vdots \\
0 & 0 & \cdots & 0 & 1 & \cdots & 0 & 0 \\
0 & 0 & \cdots & 0 & -\alpha_{i} & \cdots & 0 & 0 \\
\vdots & \vdots & \cdots & \vdots & & \cdots & \vdots & \\
0 & 0 & \cdots & 0 & 0 & \cdots & 0 & 1 \\
0 & 0 & \cdots & 0 & 0 & \cdots & 0 & -\alpha_{n}
\end{array}\right], \mathbf{B}=\left[\begin{array}{c}
0 \\
\beta_{1} \\
\vdots \\
0 \\
\beta_{i} \\
\vdots \\
0 \\
\beta_{n}
\end{array}\right] \\
\mathbf{C} & =\left[\begin{array}{cccccccc}
1 & 0 & \cdots & 0 & 0 & \cdots & 0 & 0 \\
0 & 0 & \cdots & 1 & 0 & \cdots & 0 & 0 \\
& \vdots & & & & & &
\end{array}\right]
\end{aligned}
$$

Thus, the system dynamics of a group of $n$ magnetic microbeads is linear system of order $2 n$, characterized by one input and $n$ outputs.

\section{B. System Analysis}

As mentioned, the control of a group of magnetic microbead with a sole magnetic gradient $\nabla \mathbf{b}$ belongs to the class of underactuated dynamic systems. Actually, from the system dynamics (13) one can see that $\operatorname{rank}\{\mathbf{B}\}<\operatorname{dim}(\mathbf{x})$.
Therefore, before applying a controller on the linear system (13), it would be necessary to study its controllability and observability.

Theorem 1 (Controllability): A linear system is fully controllable if and only if the rank of the controllability matrix is equal to the system order $2 n$, that is:

$$
\operatorname{rank}\left\{\mathcal{C} \triangleq\left[\begin{array}{lllll}
\mathbf{B} & \mathbf{A B} & \mathbf{A}^{2} \mathbf{B} & \ldots & \mathbf{A}^{2 n-1} \mathbf{B}
\end{array}\right]\right\}=2 n
$$

Theorem 2 (Observability): A linear system is fully observable if and only if the rank of the controllability matrix is equal to the system order $2 n$, that is:

$$
\operatorname{rank}\left\{\mathcal{O} \triangleq\left[\begin{array}{c}
\mathbf{C} \\
\mathbf{C A} \\
\vdots \\
\mathbf{C A}^{2 n-1}
\end{array}\right]\right\}=2 n
$$

From the linear system (13) with the matrices (14), it can be easily established that the state $\mathbf{x}$ is fully observable for any group of magnetic microbeads. In the following paragraph the controllability issue is addressed.

1) Case $1(n=2)$ : Let first consider the simple case of a group of $n=2$ magnetic microbeads. In such case, it is straightforward to compute the controllability matrix:

$$
\mathcal{C}_{2}=\left[\begin{array}{ccccc}
0 & \beta_{1} & \alpha_{1} \beta_{1} & \alpha_{1}{ }^{2} & \beta_{1} \\
\beta_{1} & \alpha_{1} \beta_{1} & \alpha_{1}{ }^{2} \beta_{1} & \alpha_{1}{ }^{3} & \beta_{1} \\
0 & \beta_{2} & \alpha_{2} \beta_{2} & \alpha_{2}{ }^{2} & \beta_{2} \\
\beta_{2} & \alpha_{2} \beta_{2} & \alpha_{2}{ }^{2} \beta_{2} & \alpha_{2}{ }^{3} & \beta_{2}
\end{array}\right]
$$

The maximal rank of the controllability matrix is: $\operatorname{rank}\left\{\mathcal{C}_{2}\right\}=3<4$. Especially, if the two magnetic microbeads are identical (that is $\alpha_{1}=\alpha_{2}$ and $\beta_{1}=\beta_{2}$ ) the rank of the controllability matrix fall to $\operatorname{rank}\left\{\mathcal{C}_{2}\right\}=2$. Therefore, the system of two magnetic microbeads is not fully controllable.

2) General Case: In the general case of a group of $n$ magnetic microbeads the controllability matrix is expressed:

$$
\mathcal{C}_{n}=\left[\begin{array}{ccccc}
0 & \beta_{1} & \alpha_{1} \beta_{1} & \ldots & \alpha_{1}^{n} \beta_{1} \\
\beta_{1} & \alpha_{1} \beta_{1} & \alpha_{1}^{2} \beta_{1} & \ldots & \alpha_{1}^{n+1} \beta_{1} \\
\vdots & \vdots & \vdots & \ddots & \vdots \\
0 & \beta_{n} & \alpha_{n} \beta_{n} & \ldots & \alpha_{n}^{n} \beta_{n} \\
\beta_{n} & \alpha_{n} \beta_{n} & \alpha_{n}{ }^{2} \beta_{n} & \ldots & \alpha_{n}^{n+1} \beta_{n}
\end{array}\right]
$$

The maximal rank of the above controllability matrix is:

$$
\operatorname{rank}\left\{\mathcal{C}_{n}\right\}=\frac{n}{2}-1<2 n
$$

Thus, the system is not fully controllable. In particular, for the degenerate case where all magnetic microbeads are identical we always obtain $\operatorname{rank}\left\{\mathcal{C}_{n}\right\}=2$.

3) Uncontrollable System: Basically, when a system is not completely controllable, it can be decomposed into a fully controllable subsystem and an uncontrollable subsystem in the following way

Lemma 1: Let consider an uncontrollable system such that $\operatorname{rank}\{\mathcal{C}\}=k<2 n$. For such system, there exists a state transformation such that $\chi=\mathbf{T}^{-1} \mathbf{x}=\left(\chi_{c}, \chi_{\mathrm{nc}}\right)^{T}$ (where $\chi_{c}$ is controllable, and $\chi_{\mathrm{nc}}$ uncontrollable states), that 
decomposes the system (13) in the Kalman controllability staircase form:

$$
\begin{aligned}
{\left[\begin{array}{c}
\dot{\chi}_{c} \\
\dot{\chi}_{\mathrm{nc}}
\end{array}\right] } & =\left[\begin{array}{cc}
\mathbf{A}_{c} & \mathbf{A}_{*} \\
0 & \mathbf{A}_{\mathrm{nc}}
\end{array}\right]\left[\begin{array}{c}
\chi_{c} \\
\chi_{\mathrm{nc}}
\end{array}\right]+\left[\begin{array}{c}
\mathbf{B}_{c} \\
\mathbb{0}
\end{array}\right] \mathbf{u} \\
& =\mathbf{A}_{T} \chi+\mathbf{B}_{T} \mathbf{u} \\
\mathbf{y} & =\left[\begin{array}{ll}
\mathbf{C}_{c} & \mathbf{C}_{\mathrm{nc}}
\end{array}\right]\left[\begin{array}{c}
\chi_{c} \\
\chi_{\mathrm{nc}}
\end{array}\right] \\
& =\mathbf{C}_{T} \chi
\end{aligned}
$$

with $\mathbf{A}_{T}=\mathbf{T}^{-1} \mathbf{A T}, \mathbf{B}_{T}=\mathbf{T}^{-1} \mathbf{B}$, and $\mathbf{C}_{T}=\mathbf{C T}$. Especially, $\operatorname{dim}\left(\mathbf{A}_{c}\right)=k \times k$ and the pair $\left(\mathbf{A}_{c}, \mathbf{B}_{c}\right)$ is completely controllable.

This decomposition allows to distinguish which states $\chi_{c}$ are controllable or not. The similarity transformation matrix $\mathbf{T}$ is designed from any $k$-linearly independent columns of the controllability matrix $\mathcal{C}$, from which it is appended any $(2 n-k)$ other columns such that the resulting $2 n \times 2 n$ matrix is nonsingular. Especially, it can be shown that such decomposition is not unique.

Applying this transformation to a group of $n=2$ magnetic microbeads the tuple $\left(x_{1}, x_{2}, v_{x 1}\right)^{T}$ or $\left(x_{1}, x_{2}, v_{x 2}\right)^{T}$ are the controllable subspace. By extension to the general case, the controllable subspace is $\left(x_{1}, \ldots, x_{i}, \ldots, x_{n}, v_{x j}\right)^{T}, \forall j=$ 1..n. More precisely, the position of each microbead is controllable, and only one velocity $v_{x j}$ could be controlled. For the degenerate case, the controllable states defined by $\left(x_{j}, v_{x j}\right)^{T}, \forall j=1 . . n$ shows that only one microbead can be controlled. Finally, the uncontrollable state $\chi_{\mathrm{nc}}$ equilibrium is at stability limit, as $\mathbf{A}_{\mathrm{nc}}$ have null eigenvalues. Fig. 3 shows an example of phase portrait of the dynamic system $\left(S_{i}\right)$ designed with parameters given in Table I. This result illustrates that for any initial values $v_{x i}\left(t_{0}\right)$, the velocity vanish when no input control $\mathbf{u}$ is applied. In other words, the uncontrollable states $v_{x j}$ are able to decay to origin "by themselves". Thus, each dynamic system $\left(S_{i}\right)$ is stabilizable.

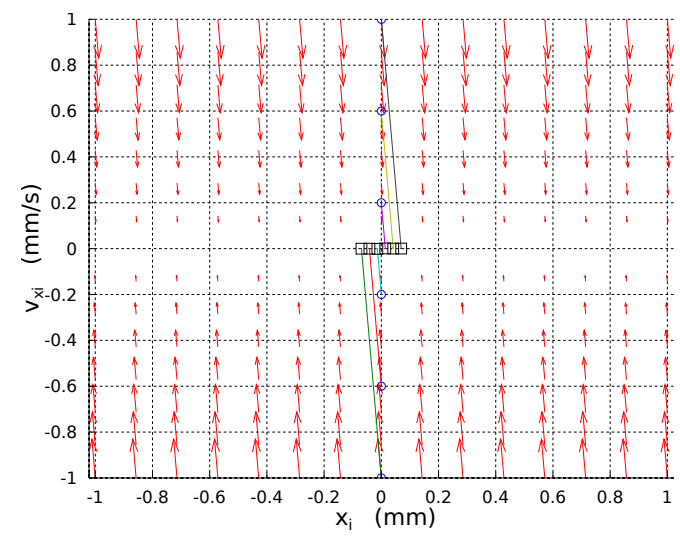

Fig. 3. Phase portrait of $\left(x_{i}, v_{x i}\right)$.

\section{Optimal Control}

The literature proposes different control schemes to actuate magnetic microbeads in a microfluidic environment, such as simple proportional-integral-derivative (PID) controller [14], predictive controller [3], [5] or adaptive backstepping controller [4]. In particular, in [5] a nonlinear model predictive controller (NMPC) is designed to control multiple millimeter-sized magnetic capsules. Commonly, predictive controller use the optimal control problem to compute the optimal trajectory over a finite-horizon. This study, propose to use the linear quadratic integral (LQI) controller. LQI control scheme is simple to implement, and has been applied in a wide range of robotic applications. Basically, LQI computes an optimal state-feedback control law, like linearquadratic regulator (LQR), with an additional integral-action on the state-feedback.

Let consider the linear system (13), to which is added the "integral state":

$$
\mathbf{w}(t)=\int_{0}^{t}\left(\mathbf{y}^{\star}(\tau)-\mathbf{y}(\tau)\right) d \tau
$$

and the following new dynamics:

$$
\dot{\mathbf{w}}=\mathbf{y}^{\star}-\mathbf{y}=\mathbf{y}^{\star}-\mathbf{C x}
$$

where $\mathbf{y}^{\star}$ is the reference input. Thus, the LQI control design address the augmented state $\mathcal{X}=(\mathbf{x}, \mathbf{w})^{T}$, that is the new system:

$$
\begin{aligned}
& \dot{\mathcal{X}}=\widetilde{\mathbf{A}} \mathcal{X}+\widetilde{\mathbf{B}} \mathbf{u} \\
& \mathbf{y}=\widetilde{\mathbf{C}} \mathcal{X}
\end{aligned}
$$

with the matrices:

$$
\widetilde{\mathbf{A}}=\left[\begin{array}{ll}
\mathbf{A} & \mathbb{0} \\
\mathbf{C} & \mathbb{0}
\end{array}\right], \widetilde{\mathbf{B}}=\left[\begin{array}{c}
\mathbf{B} \\
\mathbb{0}
\end{array}\right] \text {, and } \widetilde{\mathbf{C}}=\left[\begin{array}{ll}
\mathbf{C} & 0
\end{array}\right]
$$

Thus, the objective is to determine an optimal control law minimizing the cost functional:

$$
J(\mathbf{u})=\int_{0}^{\infty}\left\{\mathcal{X}^{T} \mathbf{Q X}+\mathbf{u}^{T} \mathbf{R u}+2 \mathcal{X}^{T} \mathbf{N u}\right\} d t
$$

where $\mathbf{Q}, \mathbf{R}$ and $\mathbf{N}$ are are symmetric, positive (semi-) definite weighting matrices, that is the LQI design parameters. This is the LQI problem, and, considering a stabilizable and detectable system, its solution yields the linear state-feedback control law:

$$
\mathbf{u}=-\left(\mathbf{R}^{-1} \widetilde{\mathbf{B}} \mathbf{P}+\mathbf{N}^{T}\right) \mathcal{X}
$$

where $\left(\mathbf{R}^{-1} \widetilde{\mathbf{B}} \mathbf{P}+\mathbf{N}^{T}\right)$ is the optimal gain matrix, with $\mathbf{P}$ the solution of the well known continuous time algebraic Riccati equation (CARE):

$$
\mathbf{P} \widetilde{\mathbf{A}}+\widetilde{\mathbf{A}}^{T} \mathbf{P}-(\mathbf{P} \widetilde{\mathbf{B}}+\mathbf{N}) \mathbf{R}^{-1}\left(\widetilde{\mathbf{B}}^{T} \mathbf{P}+\mathbf{N}^{T}\right)+\mathbf{Q}=0
$$

\section{Applications}

To apply the LQI control scheme to a group of $n$ magnetic microbeads, the first issue is to tune suitably the LQI weighting matrix $\{\mathbf{Q}, \mathbf{R}, \mathbf{N}\}$. Different strategies can be considered in their choice. Commonly, the LQI parameters have to satisfy the following conditions:

$$
\begin{aligned}
\mathbf{R} & >0 \\
\mathbf{Q} & \propto\left(\mathbf{C}^{\prime} \mathbf{C}\right) \\
\left(\mathbf{Q}-\mathbf{N R}^{-1} \mathbf{N}^{T}\right) & \geq 0
\end{aligned}
$$


From these rules of thumb, suitable weighting matrices for the dynamic system (13) could be obtained according the following methodology:

$$
\begin{aligned}
& \mathbf{R}=\gamma, \quad \forall \gamma \in \mathbb{R}^{+} \\
& \mathbf{Q}=\operatorname{diag}(\mathbf{q}), \text { with } \mathbf{q}=\left(q_{1} \gamma, 0, \cdots, q_{i} \gamma, 0 \cdots, q_{n} \gamma, 0\right), \\
& \mathbf{N} \ll \sqrt{\gamma \mathbf{q}}
\end{aligned}
$$

where $\forall q_{i}>0, i=1 . . n$ could be designed from the ratio between $\alpha_{i}(10)$.

As example, Fig. 4 illustrates the optimal stabilization of three magnetic microbeads of radii $r_{1}=500 \mu \mathrm{m}, r_{2}=$ $750 \mu \mathrm{m}$ and $r_{3}=1000 \mu \mathrm{m}$. Starting from the initial state $\mathbf{x}\left(t_{0}\right)=(1 \mathrm{~mm}, 0,2.5 \mathrm{~mm}, 0,4 \mathrm{~mm}, 0)^{T}$ they reach the desired output $\mathbf{y}^{\star}=(2 \mathrm{~mm}, 5 \mathrm{~mm}, 8 \mathrm{~mm})^{T}$ in a time duration of $1 \mathrm{~s}$. Different LQI parameters are used considering the above methodology (31) with:

$$
\begin{aligned}
\mathbf{R} & =\gamma=1 \\
\mathbf{q} & =\left(\frac{\gamma}{q_{0}}, 0, \frac{1.5 \gamma}{q_{0}}, 0, \frac{2 \gamma}{q_{0}}\right), \quad \forall q_{0}>0 \\
\mathbf{N} & =0
\end{aligned}
$$

The choice of the tuning parameters significantly influence the system's response and positioning performances $\mathbf{y}^{\star}$. As it can be seen, the value of $q_{0}=20$ leads to an optimum LQI parameterization with small overshot and faster settling time.

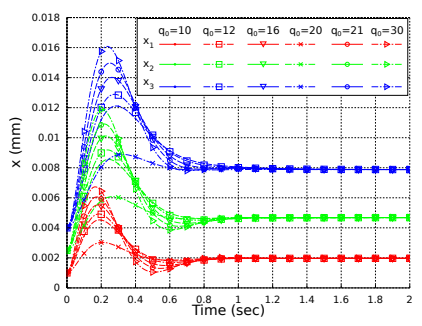

(a)

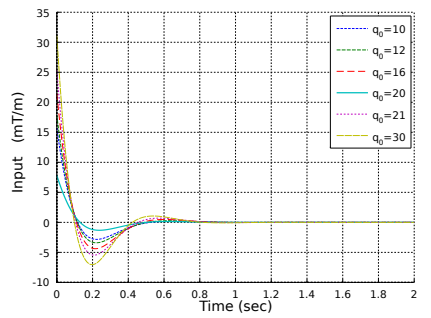

(b)
Fig. 4. Optimal stabilization of the three microbeads positions $\left(x_{10}=\right.$ $1 \mathrm{~mm}, x_{20}=2.5 \mathrm{~mm}$ and $x_{10}=4 \mathrm{~mm}$ ) to the desired state $\mathbf{y}^{\star}=$ $(2 \mathrm{~mm}, 5 \mathrm{~mm}, 8 \mathrm{~mm})^{T}$ with different LQI parameters:: (a) the state position and (b) the control input $\mathbf{u}$.

Similarly, Fig.5(a) shows the 2D stabilization of two magnetic microbeads of radii $r_{1}=500 \mu \mathrm{m}$ and $r_{2}=$ $1000 \mu \mathrm{m}$. Once again, the dynamic behavior of the magnetic microbeads is impacted by the choice of the LQI parameters. These results exhibit the feasibility to control several magnetic microbeads using a sole magnetic gradient $\nabla \mathbf{b}$.

\section{EXPERIMENTAL RESULTS}

\section{A. Experimental Setup}

To validate experimentally the findings of model simulations, an experimental setup has been specifically developed by Aeon Scientific. The system consists of three nested sets of Maxwell coils and one nested set of Helmholtz coils [15], and is illustrated in Fig.6(a). Such arrangement allows generating a constant-gradient magnetic field pointing in $x$,

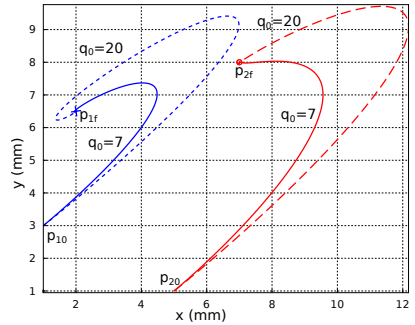

(a)

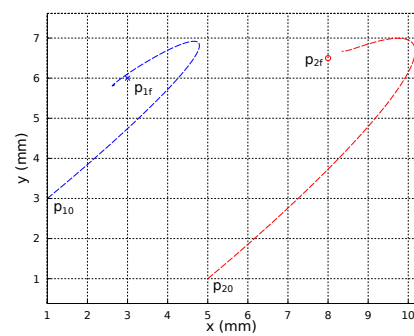

(b)
Fig. 5. Optimal stabilization of the $2 \mathrm{D}$ positions $\left(\mathbf{p}_{10}=(1,3) \mathrm{mm}\right.$ and $\left.\mathbf{p}_{20}=(5,1) \mathrm{mm}\right)$ of two magnetic microbeads to the desired state: (a) $\mathbf{y}^{\star}=(2,6.5,7,8)^{T} \mathrm{~mm}$ with different LQI parameters and (b) $\mathbf{y}^{\star}=$ $(3,6,8,6.5)^{T} \mathrm{~mm}$ with different LQI parameters.

$y$, and $z$-axis directions. The generated magnetic gradient is saturated to $\nabla \mathbf{b}_{\max }=40 \mathrm{mT} / \mathrm{m}$ to be compatible with clinical MRI scanner. Magnetic gradient forces will thus be exerted on the different magnetic microbeads that are placed inside a microfluidic environment Fig.6(b). This microfluidic environment is filled with an aqueous solution of $50 \%$ water$50 \%$ glycerol that is closed to the blood viscosity. The Table I summarizes the relevant experimental parameters set used for two case study: position control of two microbeads along identical and opposite directions.

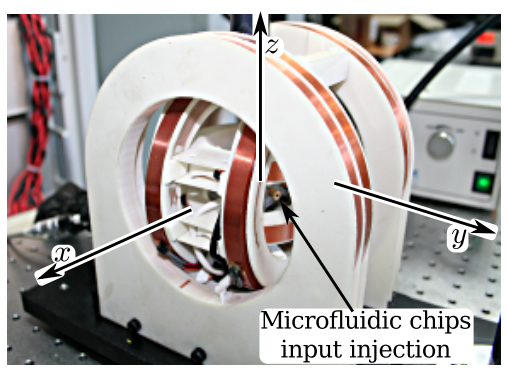

(a)

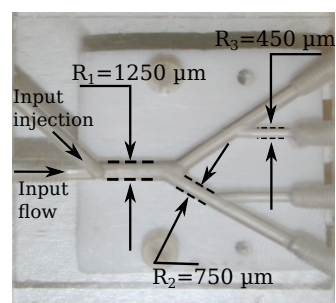

(b)
Fig. 6. Experimental setup: (a) 3D Maxwell-Helmholtz coils and (b) a W-shaped microfluidic arterial bifurcation chip.

TABLE I

MODEL PARAMETERS

\begin{tabular}{c|cl} 
Parameters & \multicolumn{2}{|c}{ Values } \\
\hline$M_{\text {sat }}$ & $1.23 \times 10^{6}$ & $(\mathrm{~A} / \mathrm{m})$ \\
$\rho$ & 7500 & $\left(\mathrm{~kg} / \mathrm{m}^{3}\right)$ \\
$r_{0}$ & 300 & $(\mu \mathrm{m})$ \\
$\eta_{f}$ & 4 & $(\mathrm{mPa})$ \\
\hline
\end{tabular}

\section{B. Control of two magnetic microbeads in the same direction}

We evaluated experimentally the controllability and stability of two microbeads motion along a similar direction ( $x$-axis) in the mother branch of the $\mathrm{W}$-shaped microfluidic arterial bifurcation phantom. As shown in Fig. 7(a), the initial distance separating the microbeads is settled to $S=7 \mathrm{~mm}$ in order to counteract the attractive forces. Each microbead faces the drag force of the constant flow $v=21 \mathrm{~mm} . \mathrm{s}^{-1}$ and experiences the same magnetic force $F_{m 1 x}=F_{m 2 x}$ since both 
microbeads are subjected to the same input: the magnetic gradient field $\nabla \mathbf{b}$. For this reason, the linear quadratic integral control is applied to control the position of one microbead while the other one follows its motion in order to reach the desired state and stay there. In the first trial, two similar magnetic microbeads $\left(R_{1}=R_{2}=250 \mu \mathrm{m}\right)$ were chosen. As shown in the Fig. 7(b), the initial positions of the microbeads 1,2 are $9.2 \mathrm{~mm}, 2.2 \mathrm{~mm}$ respectively. They move with a maximum velocity motion of $0.01 \mathrm{~mm} / \mathrm{s}$ against the flow from their initial positions until to be stabilized on final positions at $P_{1}=5.7 \mathrm{~mm}, P_{2}=-1.6 \mathrm{~mm}$ (Fig. 7(c)). The final position errors $\varepsilon_{\text {pos } 1}$ and $\varepsilon_{\text {pos } 2}$ are respectively $50 \mu \mathrm{m}$ and $250 \mu \mathrm{m}$ as seen in Fig. 8(a). In the second trial, two different magnetic microbeads $\left(R_{1}=250 \mu \mathrm{m}\right.$ and $\left.R_{2}=500 \mu \mathrm{m}\right)$ were chosen. The experimental results are shown in in Fig. 8(b). As we can see similar stability and positioning performances are obtained.

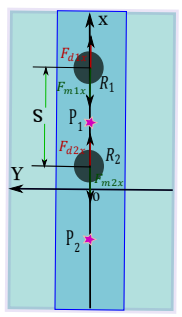

(A)

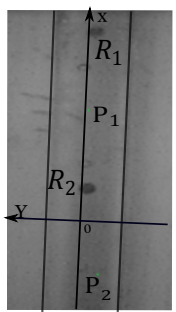

(B)

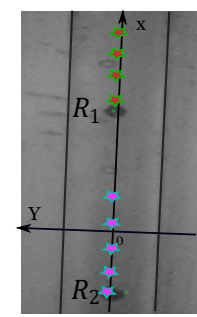

(C)
Fig. 7. Motion control of two microbeads along the same $x$-axis. (a): Experimental conditions, (b): microbeads at their initial positions, and (c): microbeads at the desired positions in the mother branch of the microfluidic arterial phantom.

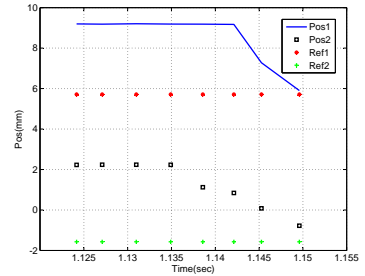

(a)

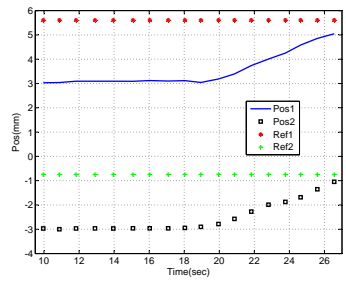

(b)
Fig. 8. Experimentally stabilization of the two microbeads positions at the desired state: (a) the microbeads sizes ratio is equal 1 (b) the microbeads sizes ratio is equal 2 .

\section{Control of two magnetic microbeads in opposite direction}

We evaluated experimentally the controllability and stability of two microbeads moving along an opposite direction $(x$-axis) in the mother branch of the W-shaped microfluidic arterial bifurcation phantom. Two different magnetic microbeads $\left(R_{1}=250 \mu \mathrm{m}\right.$ and $R_{2}=500 \mu \mathrm{m}$ ) were chosen for the experience. As shown in Fig. 9(a), the initial distance separating the microbeads is settled to $S=6 \mathrm{~mm}$ in order to counteract the attractive forces. Each microbead faces different drag force $F_{d 1 x} \neq F_{d 2 x}$ and magnetic force $F_{m 1 x}$ $\neq F_{m 2 x}$ at constant velocity flow $v=10 \mathrm{~mm} . \mathrm{s}^{-1}$. As shown in the Fig. 9(b), the initial positions of the microbeads 1,2 are $3.2 \mathrm{~mm},-2.8 \mathrm{~mm}$ respectively and final positions of the microbeads 1,2 are $P_{1}=5.8 \mathrm{~mm}, P_{2}=-5.8 \mathrm{~mm}$ (Fig. 9(c)), respectively. Compared to previous results, we noticed that the optimal LQI controller ensures good performances in terms of tracking and positioning errors even if different positive and negative drag forces are experienced by the microbeads $\left(\varepsilon_{\text {pos } 1}=25 \mu \mathrm{m}\right.$ and $\varepsilon_{\text {pos } 2}=18 \mu \mathrm{m}$ as seen in Fig. 10).

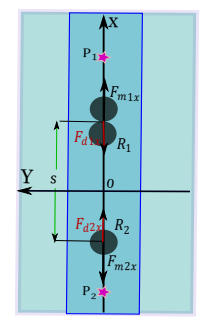

(A)

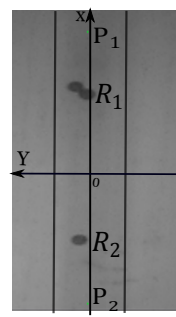

(B)

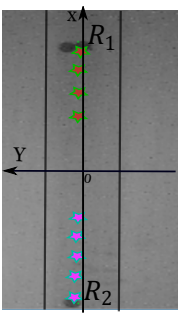

(C)
Fig. 9. Motion control of two microbeads in opposite direction along the $x$ axis. (a): Experimental conditions,(b): microbeads at their initial positions, and (c): microbeads at the desired positions in the mother branch of the microfluidic arterial phantom.

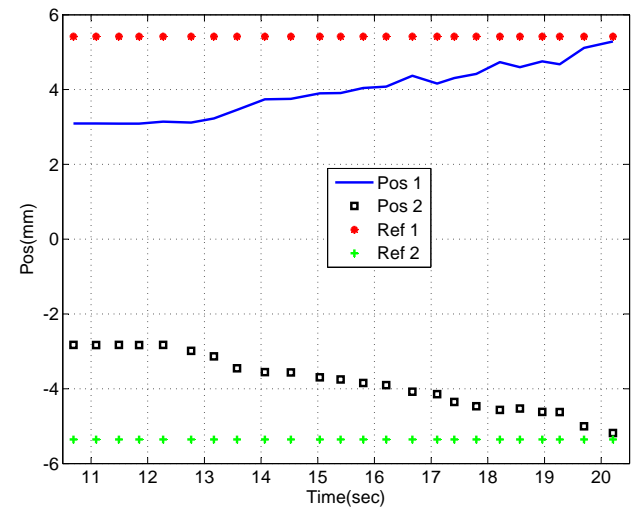

Fig. 10. Experimentally stabilization of the two microbeads positions at the desired state, the microbeads sizes ratio is equal 2 .

\section{CONCLUSION}

This paper described the theoretical formulation of an optimal control strategy for navigation of multiple magnetic microbeads for future drug targeting applications. From the developed dynamic model and state representation of a group of magnetic microbeads, controllability and observability conditions are formulated. Simulations demonstrated necessary stability conditions to control independently each magnetic microbead with the same magnetic gradient field in order to reach the desired positions. A linear quadratic with integral action control (LQI) has been synthesized and implemented experimentally in a electromagnetic system. The preliminary experiments carried out in microfluidic artery vessels demonstrated successfully that it was possible to stabilize at the desired positions two geometrically similar 
and/or dissimilar different magnetic microbeads in a microfluidic phantom. Further developments will be carried out in order to take into account the magnetic interaction between the microbeads, and then, to extend it to an aggregate of magnetic microbeads navigating in multiple bifurcations.

\section{REFERENCES}

[1] P. Pouponneau, J.-C. Leroux, G. Soulez, L. Gaboury, and S. Martel, "Co-encapsulation of magnetic nanoparticles and doxorubicin into biodegradable microcarriers for deep tissue targeting by vascular mri navigation," Biomaterials, no. 32, pp. 3481-3486, 2011.

[2] P. Vartholomeos, M. R. Akhavan-Sharif, and P. E. Dupont, "Motion planning for multiple millimeter-scale magnetic capsules in a fluid environment," in Robotics and Automation (ICRA), 2012 IEEE International Conference on. IEEE, 2012, pp. 1927-1932.

[3] K. Belharet, D. Folio, and A. Ferreira, "Three-dimensional controlled motion of a microrobot using magnetic gradients," Advanced Robotics, vol. 25, no. 8, pp. 1069-1083, 2011.

[4] L. Arcese, M. Fruchard, and A. Ferreira, "Adaptive controller and observer for a magnetic microrobot," IEEE Trans. Robot., vol. PP, no. 99 , pp. $1-8,2013$.

[5] A. Eqtami, O. Felfoul, and P. E. Dupont, "Mri-powered closed-loop control for multiple magnetic capsules," in IEEE/RSJ Int. Conf. on Intell. Robots and Syst. (IROS). Chicago, Il, USA: IEEE, 2014, pp. 3536-3542.

[6] E. Diller, S. Floyd, C. Pawashe, and M. Sitti, "Control of multiple heterogeneous magnetic microrobots in two dimensions on nonspecialized surfaces," Robotics, IEEE Transactions on, vol. 28, no. 1, pp. 172-182, 2012.

[7] J. Shin, K. Nonami, D. Fujiwara, and K. Hazawa, "Model-based optimal attitude and positioning control of small-scale unmanned helicopter," Robotica, vol. 23, no. 01, pp. 51-63, 2005.

[8] K. Seto, D. FUJI, H. HIRAMATHU, and T. Watanabe, "Motion and vibration control of three dimensional flexible shaking table using lqi control approach," in Proceedings of American Control Conference, 2002, vol. 4. IEEE, 2002, pp. 3040-3045.

[9] T. Tsujimura and T. Manabe, "Electromagnetic system navigating tunneling robots," Robotics and Autonomous Systems, vol. 40, no. 4, pp. 229-238, 2002.

[10] G. G. Stokes, On the effect of the internal friction of fluids on the motion of pendulums. Pitt Press, 1851, vol. 9.

[11] P. Vartholomeos and C. Mavroidis, "In silico studies of magnetic microparticle aggregations in fluid environments for MRI-guided drug delivery," IEEE Trans. Biomed. Eng., vol. 59, no. 11, pp. 3028-3038, 2012.

[12] R. Schill et al., "General relation for the vector magnetic field of a circular current loop: a closer look," IEEE Trans. Magn., vol. 39, no. 2, pp. 961-967, 2003.

[13] D. Villani, "An analytic solution for the force between two magnetic dipoles," Magnetic and electrical Separation, vol. 9, pp. 39-52, 1998.

[14] S. Tamaz, R. Gourdeau, A. Chanu, J.-B. Mathieu, and S. Martel, "Realtime MRI-based control of a ferromagnetic core for endovascular navigation," IEEE Trans. Bio-Med. Eng., vol. 55, no. 7, pp. 1854$1863,2008$.

[15] K. Belharet, D. Folio, and A. Ferreira, "Control of a magnetic microrobot navigating in microfluidic arterial bifurcations through pulsatile and viscous flow," in IEEE/RSJ Int. Conf. on Intell. Robots and Syst. (IROS). IEEE, 2012, pp. 2559-2564. 